\title{
Secador solar tipo túnel con microclima auto controlado para Café (Coffea arabica) Honey de alto valor en taza
}

\section{Tunnel type solar dryer with self-controlled microclimate for Coffee (Coffea arabica) Honey of high value in cup}

\author{
Dávila-Guamuro, Jhon [(D) 0000-000I-6201-6663]'; Llanos-Pérez, Jossy [(D) 0000-0002-503I-663I]I; Cabanillas- \\ Pardo, Lenin [D 0000-0002-1059-8064]। \\ 'Prolnnóvate, Perú \\ $\triangle$ jhond9013@gmail.com
}

Recibido: 27/10/2021;

Aceptado: 29/1I/2021;

Publicado: 20/0I/2022

Resumen: Perú a nivel mundial es referente en comercialización de cafés especiales y de taza limpia aplicando comercio justo y tiene la variedad Honey cuyas características organolépticas son de alta demanda. El objetivo fue diseñar y construir un secador solar tipo túnel con microclima auto controlado para cafés Honey de alto valor en taza en la provincia de Moyobamba. Para ello se evaluaron diferentes diseños que incorporen elementos electrónicos con capacidad de calefacción de aire utilizando energía solar y extractores de humedad con estructuras metálicas para remoción de forma automática cada 20 min en el que se hicieron experimentos de secado a diferentes temperatura y tiempo de granos de café Honey. Se construyó un prototipo de secador en forma geométrica de bóveda como elemento constructivo superficial, con bandejas para secado del café a temperaturas máximas de $35 \mathrm{C}^{\circ}$ con remoción del grano cada 20 min en cuyas instalaciones se logró obtener granos de café Honey con humedad entre $12 \%$ a $14 \%$ cuyas muestras luego de procesadas obtuvieron en promedio 82 puntos en taza. El dispositivo ha cumplido su propósito de secado del café Honey en condiciones asépticas, automáticas y controladas para obtener café Honey con calidad de taza limpia.

Palabras clave: calidad; prototipo; secado; sensorial; taza limpia

Abstract: Peru is a world benchmark in the commercialization of specialty and clean cup coffees applying fair trade and has the Honey variety whose organoleptic characteristics make it in high demand. The objective was to design and build a tunnel-type solar dryer with a self-controlled microclimate for high value cup Honey coffees in the province of Moyobamba. For this, we evaluated different designs that incorporate electronic elements with air heating capacity using solar energy and moisture extractors with metal structures for automatic removal every 20 minutes in which we made drying experiments at different temperature and time with grains of Honey coffee. We built a dryer prototype in a geometric vault shape as a surface constructive element; with trays for drying the coffee at maximum temperatures of $35^{\circ} \mathrm{C}$ with removal of the bean every 20 minutes in whose facilities has been possible to obtain Honey coffee beans with humidity between $12 \%$ to $14 \%$ whose samples after being processed obtained an average of 82 points in cup. The device has served its purpose of drying Honey coffee in aseptic, automatic and controlled conditions to obtain Honey coffee with clean cup quality.

Keywords: quality; prototype; drying; sensory; clean cup

Cómo citar / Citation: Dávila-Guamuro, J., Llanos-Pérez, J., \& Cabanillas-Pardo, L. (2022). Secador solar tipo túnel con microclima auto controlado para Café (Coffea arabica) Honey de alto valor en taza. Revista agrotecnológica amazónica, 2(I), e227. https://doi.org//0.5I252/raa.v2il.227 


\section{Introducción}

PERÚ es un referente a nivel mundial de cafés especiales, además ocupa el 2 do lugar a nivel mundial como productor y exportador de café orgánico y es el primer proveedor de EE.UU. de café con el sello de Fair Trade (comercio justo) abarcando el 25\% del nicho de mercado (Estevez et al., 2018), se ubica entre los Top Ten como productor/exportador de café a nivel mundial según la Organización Internacional del Café (ICO) (International Coffee Organization, 202I). La exportación de Café Grano en el 2017 alcanzó los U\$ 694 millones a un precio promedio de U\$ 2.89 kilo, las divisas recibidas por el café peruano representaron un incremento de $2,27 \%$ a lo registrado en el mismo período de 2016 y su consumo per cápita es de 850 gr., habiendo incrementado en un 30\% en los últimos 4 años (Díaz Vargas \& Carmen Willems, 2017). Se estima que el negocio del café siga la misma ruta al crecimiento de la gastronomía (Arias Escobar et al., 2017).

De la misma manera la demanda por cafés especiales como el Honey ha crecido en un II\% los dos últimos años, nuestro país exportó 10 conteiner de café el año 2016, frente a una demanda dando motivación de ofertar este tipo café; según fuentes Wall Stree: Starbucks en convenio con NESTLE tienen una oferta de alrededor de 2 millones de quintales de cafés especiales (entre ellos café Honey) con tasas superiores a 83 puntos SCAA.

Según las informaciones estadísticas de mercado que el consumo de cafés con tasas superiores a 84 puntos va en aumento en un 10\% del respecto del año anterior (International Coffee Organization, 202I). El año 2016 nuestro país exportó 5,198,9lI, y el año 2017 se exportó más de 6 millones de quintales de café, el cual los destinos son USA y EUROPA, del cual el mercado Alemán es el segundo después USA en preferencia, por lo que favorece a la economía nacional con representación en valor de 800 millones de dólares, el cual motiva cada día a nuestro asociados en mejorar su producción y calidad sensorial de grano.

Apecam es una organización de 207 agricultores asociados, ubicados en una de las mejores zonas cafetaleras del Valle del Alto Mayo con plantaciones que se ubican entre los 1200 a 1800 msnm, que se dedican a la producción de café. Acopian y comercializan cafés certificados o convencionales de acuerdo al requerimiento. Cuenta con certificaciones de comercio justo y orgánico (Márquez Romero et al., 2016), sin embargo, los problemas en cuanto a infraestructura para los diferentes procesos y especialmente el de secado, impide escalar la producción de cafés especiales en mayores volúmenes repercutiendo en su productividad. Su proceso de secado hace uso intensivo y excesivo de mano de obra.

El proceso convencional usado, que requiere entre 8 a 10 días, para obtener café Honey consiste en rebalsar el café cerezo, despulpar y secarlo a sol al aire libre en mantas de polipropileno, punto crítico pues $60 \%$ de la producción se deteriora, esencialmente la calidad sensorial que llega solo a 84 siendo la exigencia superar los 85 puntos. Para ello se han evaluado tecnologías de secado que usan dispositivos electro mecánicos y electrónicos para la remoción y medición de la humedad del grano como los de Barrera et al. (2019), Chunshan et al. (2016) y Guevara-Sánchez et al. (2019), así como la incorporación de ventilación controlada de aire caliente en los diferentes estadíos del proceso como Chunshan et al. (2016).

Los productores no cuentan con protocolos con estándares definidos para el secado de cafés proveniente de procesos Honey repercutiendo la calidad sensorial (Rodriguez et al., 2020), 
lo realizan de forma convencional aprovechando los rayos solares, cuya radiación es indistinto cada día (Kaveh et al., 202I), por ejemplo los cafés cultivados en las partes bajas ( $800 \mathrm{msnm}$ I $100 \mathrm{msnm}$ ) el secado de grano es más rápido (entre 8 a 12 días) que el café cultivado en las partes de mayor altitud (mayor a $1100 \mathrm{msnm}$ ) el secado es más prolongada (mayor a 12 días) por cual el grano es de mayor consistencia y densidad, por lo que se requiere estandarizar el proceso para cada nivel altitudinal (Barrera et al., 2019)(Guevara-Sánchez et al., 2019). Por otro lado, el mucilago representa $15 \%$ del peso fresco de café cerezo, en el despulpado se elimina cerca del 50\%; sin embargo, aún es bastante mucílago que se queda en el grano, que si no llevamos un manejo eficiente de secado este tiende a fermentarse si no se seca en condiciones de temperatura y ventilación de manera correcta.

Al no manejar de forma eficiente los procesos de post cosecha principalmente el secado para el caso de cafés Honey (Rodriguez et al., 2020), las potencialidades de calidad de grano se ven afectadas y disminuidas, no logrando superar los 84 puntos de calidad de taza (Specialty Coffee Association, 2003). El proceso de secado sin control de tiempo, ventilación y temperatura (Larasati et al., 2018), dificulta a la cooperativa obtener cafés con altas valoraciones en taza (calidad sensorial) y atributos diferenciados catalogados como de especialidad (Borém et al., 2013), el cual limita su acceso a nichos de mercado con mayores volúmenes al esperado para lograr un poder de negociación.

El objetivo del proyecto es diseñar y construir un prototipo de secador solar tipo túnel con microclima auto controlado para mejorar el proceso de secado con tecnología de termocontrol y aprovechamiento térmico para obtener calidad de los granos para cafés Honey de alta calidad en taza en la provincia de Moyobamba.

\section{Materiales y métodos}

La APECAM, cuenta con un centro de acopio de café de más de $1,000 \mathrm{~m} 2$, con infraestructura de secado integrada por guardiolas rotatorias para los cafés lavados certificados y convencionales producidos por sus asociados ubicado en el distrito de Soritor, provincia de Moyobamba en la región San Martín. Así mismo, cuentan con un camión de 10 TM con el que organizan el acopio desde campo hacia el almacén central, y personal técnico especializado. Así mismo sus asociados cuentan en gran porcentaje con infraestructura de beneficio y post cosecha para procesos de café lavado.

\section{Construcción del prototipo}

Para la construcción del prototipo se evaluaron varias alternativas de secado basado en energía solar (Larasati et al., 20I8) y colección de aire temperado (Adonis \& Khan, 2004) como también el de Prada et al. (2019). Así mismo revisamos modelados dinámicos de secadores de granos de café (Zuluaga-Bedoya \& Gómez, 2015) y el clasificador basado en contenido de humedad de (Alibayan et al., 2019).

Inicialmente se ha realizado la limpieza de $48 \mathrm{~m} 2$ de terreno, se instalaron y ensamblaron una estructura metálica y las bandejas de secado donde se esparcirá el café Honey para secado. También se incluyeron obras de concreto simple y la habilitación de un techo con la colocación de planchas de prolipropileno unidas con $\mathrm{H}$ y utilizando silicona para pegar las uniones y quedar selladas según la ¡Error! No se encuentra el origen de la referencia.. 
El diseño planteado es con tecnología tipo parabólico y con sistema de ductos de entrada de aire y evacuación de humedad y con el sistema de remoción automatizado (Bolaños et al., 2018).

Dentro de las actividades del proyecto se ha programado la mejora y acondicionamiento de áreas de trabajo para la construcción del dispositivo.

El sistema capta el calor térmico del sol que se almacena en el invernadero con rangos de temperatura de entre 30 a $39^{\circ} \mathrm{C}$ todo el día, a través de un sistema de ductos elimina la humedad resultante que se miden mediante sensores de humedad relativa del entorno del secador $y$ temperatura para activar el colector solar del aire.

\section{Recolección transporte y almacenamiento de los frutos}

La recolección se realizó en los caseríos Los Claveles y Jericó del distrito de Soritor, dos muestras por zona. Se determinó la madurez según su genotipo y la intensidad de la coloración separando manualmente los frutos maduros de los verdes, pintones, sobre maduros y secos. Para la movilización no se arrumaron los frutos por más de tres horas, se evitó la presencia de animales y limpieza del lugar eliminando contaminantes físicos y químicos. Evitamos que el café cosechado se contamine con hongos o bacterias (contaminantes biológicos) impidiendo contacto con el suelo. Las personas encargadas del procesamiento se encuentran en buen estado de salud.

\section{Clasificación del café cosechado}

Se clasificaron por densidad separando frutos maduros del resto utilizando tanques con agua limpia donde se deposita el café cosechado, se agita hasta que los flotantes (frutos vanos, tiernos, secos, basura, hojas y palos) sean retirados manualmente usando un tamiz. Continuamos con el proceso de beneficio con los frutos maduros que, por su densidad, quedaron asentados en el fondo.

\section{Beneficiado, secado y almacenamiento del café}

Utilizamos el beneficio semi húmedo - Honey, los frutos maduros son despulpados y secados con el mucílago adherido al grano (Rodriguez et al., 2020). Utilizamos el prototipo construido, esparciendo los granos en capas delgadas de $3 \mathrm{~cm}$ y removiendo cada 20 minutos a temperaturas máximas de $35{ }^{\circ} \mathrm{C}$ y mínimas de $20{ }^{\circ} \mathrm{C}$ controladas con colectores de aire a base de energía solar evitando mezclar granos con diferente contenido de humedad. Se realizó monitoreo con el software del prototipo (da Costa et al., 202I). La clasificación antes del despulpado y el secado son las etapas importantes en la calidad final para que presente atributos sensoriales dulces y frutales, conservando el nivel de acidez para ser comercialmente bien valorado. Las muestras humedad entre $12 \%$ a $14 \%$ se dejaron en reposo en parihuelas de madera separadas de la pared, con una altura entre 10 y $15 \mathrm{~cm}$ del suelo durante una semana en una bodega seca y ventilada, con temperatura menor a $20^{\circ} \mathrm{C}$ y humedad relativa del $65 \%$ al $70 \%$ para evitar rehumedecimiento del producto (Sotelo-Valer et al., 2020). El almacén de café estuvo separado del centro de beneficio y cerca de la bodega se evitaron olores. 


\section{Resultados y discusión}

\section{Construcción del prototipo}

Figura I. Diseño en 3D con sus bandejas, sistemas de ductos de entradas de aire, colector solar y sistema de extracción de humedad.

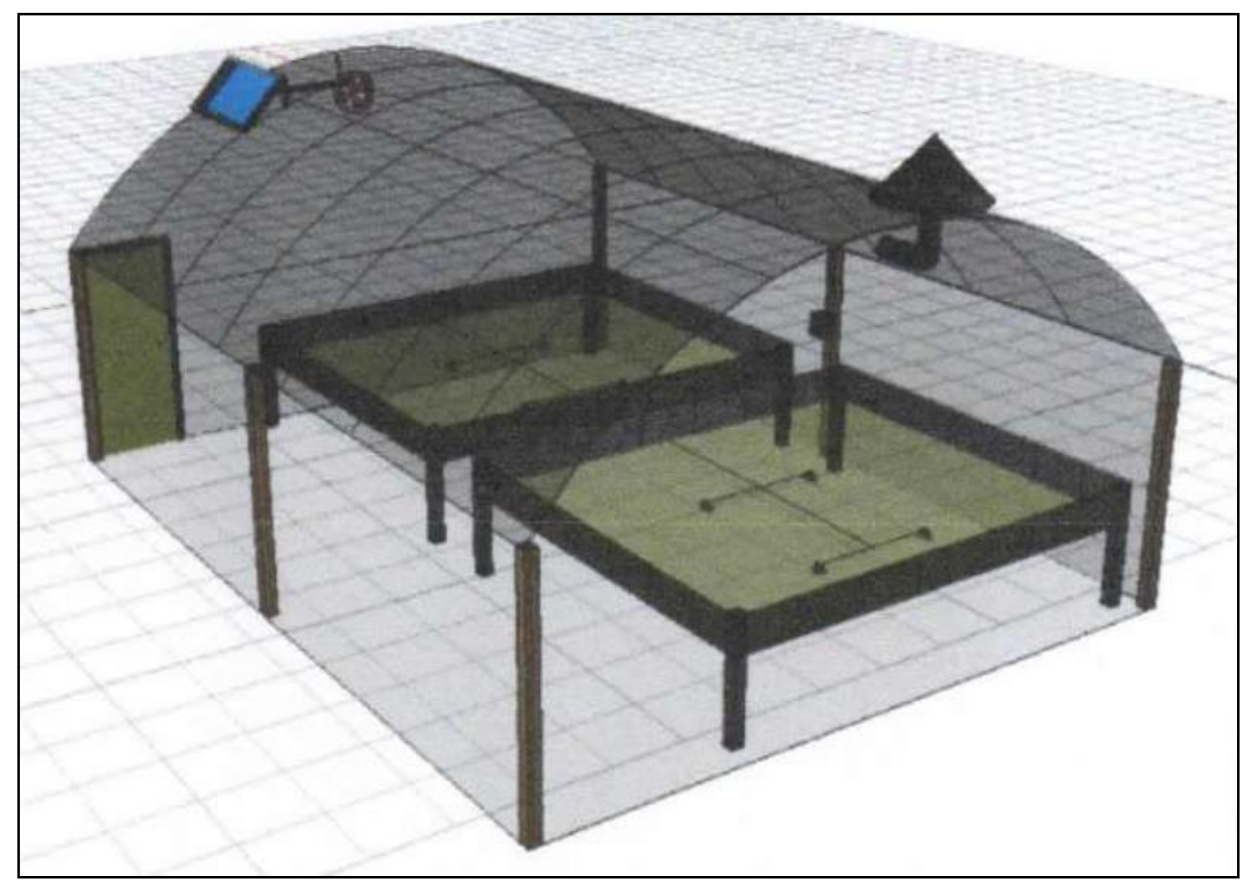

Figura 2. Diseño vista 3D del sistema de remoción lineal activado con panel de control.

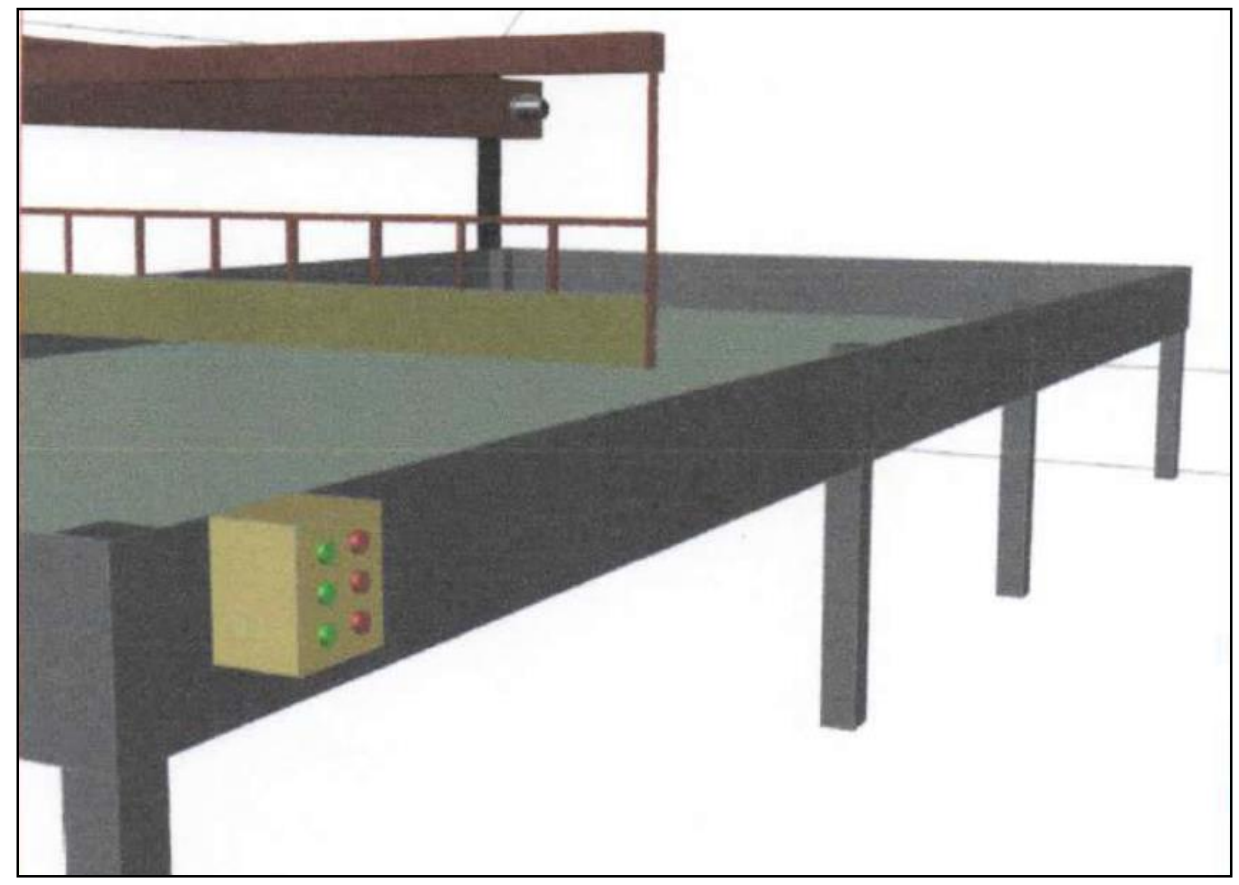


Figura 3. Diseño vista 3D donde se divisa el prototipo con su sistema de remoción lineal, activado panel de control y sistema de colector solar.

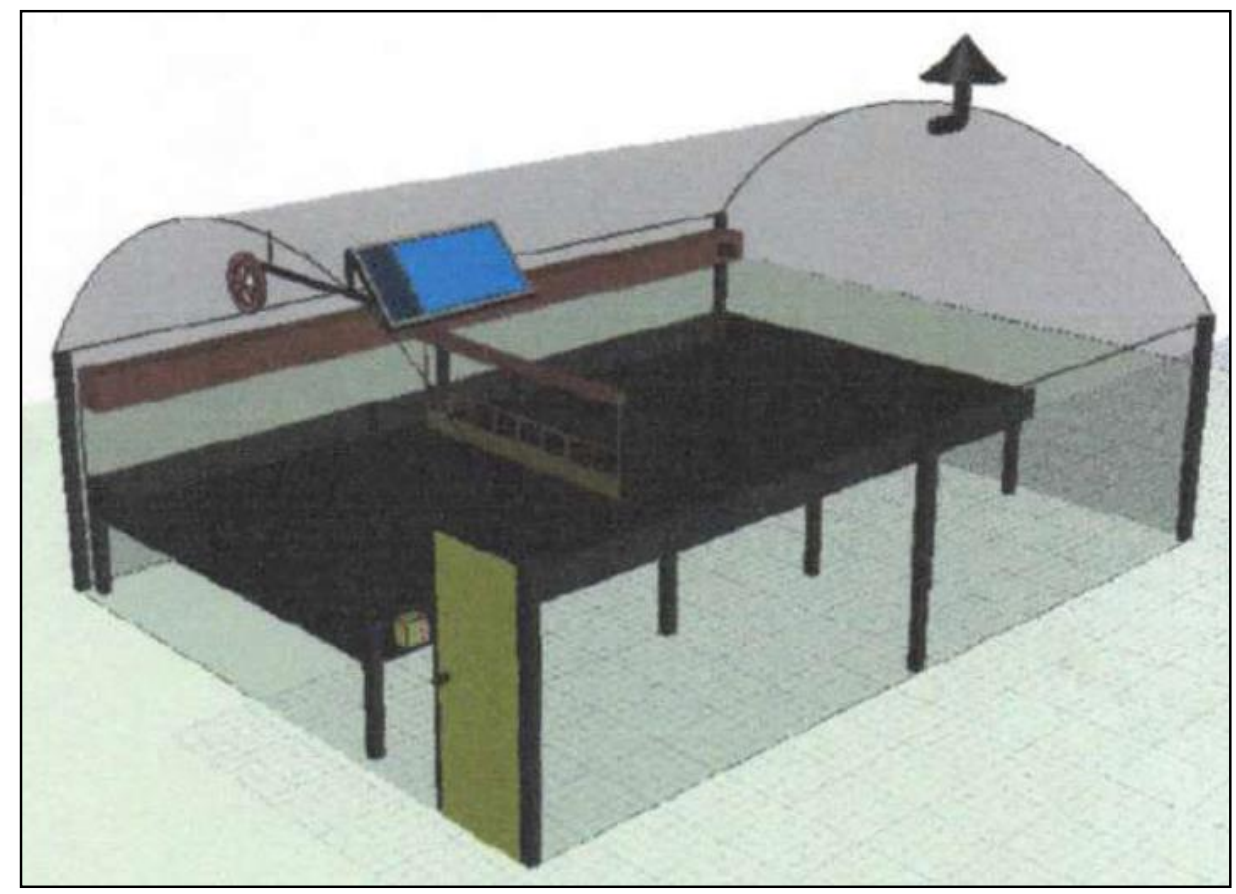

\section{Efecto sobre la calidad sensorial}

Se realizaron el análisis de calidad física y organoléptica de 40 muestras de café Honey; los resultados se presentan en la siguiente tabla.

Tabla I. Resultados de la evaluación sensorial, de rendimiento y humedad de 40 muestras de café Honey secadas con el prototipo.

\begin{tabular}{|c|c|c|c|c|c|c|c|c|c|c|c|c|}
\hline \multirow{3}{*}{ Código } & \multicolumn{6}{|c|}{ Zona cafetalera Los Claveles } & \multicolumn{6}{|c|}{ Zona cafetalera Jericó } \\
\hline & \multicolumn{3}{|c|}{$\mathrm{Cl}$} & \multicolumn{3}{|c|}{$\mathrm{C2}$} & \multicolumn{3}{|c|}{$\mathbf{C l}$} & \multicolumn{3}{|c|}{$\mathrm{C2}$} \\
\hline & PT & $\mathbf{R}$ & $\mathbf{H}$ & PT & $\mathbf{R}$ & $\mathbf{H}$ & PT & $\mathbf{R}$ & $\mathbf{H}$ & PT & $\mathbf{R}$ & $\mathbf{H}$ \\
\hline $\mathrm{I}$ & 82.00 & 73.00 & 13.50 & 81.75 & 71.00 & 13.20 & 82.75 & 71.61 & 14.20 & 82.00 & 76.60 & 14.40 \\
\hline 2 & 82.00 & 74.00 & 13.00 & 82.25 & 69.00 & 13.80 & 82.50 & 71.66 & 14.50 & 82.25 & 75.00 & 13.90 \\
\hline 3 & 82.25 & 74.00 & 13.80 & 81.75 & 70.00 & 13.40 & 82.75 & 71.66 & 14.20 & 82.25 & 75.00 & 14. \\
\hline 4 & 81.50 & 73.00 & 13.60 & 81.75 & 69.33 & 13.20 & 82.50 & 71.66 & 14.20 & 82.00 & 79.00 & 13.80 \\
\hline 5 & 81.75 & 72.00 & 13.60 & 82.00 & 70.00 & 13.70 & 82.75 & 71.66 & 13.70 & 82.25 & 75.66 & 13.80 \\
\hline 6 & 82.25 & 74.00 & 13.60 & 81.50 & 69.33 & 13.70 & 82.75 & 70.66 & 14.10 & 82.25 & 75.00 & 14.70 \\
\hline 7 & 82.00 & 73.00 & 13.40 & 82.50 & 69.33 & 14.00 & 82.25 & 70.00 & 14.70 & 81.75 & 74.66 & 14.40 \\
\hline 8 & 82.00 & 74.00 & 14.00 & 82.25 & 71.00 & 13.60 & 82.25 & 72.00 & 14.40 & 82.25 & 75.33 & 14.30 \\
\hline 9 & 82.00 & 74.00 & 13.90 & 82.00 & 72.68 & 12.80 & 82.25 & 71.66 & 14.00 & 82.00 & 76.00 & 14.10 \\
\hline 10 & 82.00 & 73.00 & 14.00 & 81.50 & 72.33 & 12.70 & 82.75 & 72.00 & 12.30 & 82.00 & 74.66 & 14.10 \\
\hline Prom. & 81,98 & 73,40 & 13,64 & 81,93 & 70,40 & $|3,4|$ & 82,55 & 71,46 & 14,03 & 82,10 & 75,69 & 14,16 \\
\hline
\end{tabular}

Dónde: $\mathrm{PT}=$ Puntos en tasa, $\mathrm{R}=$ rendimiento, $\mathrm{H}=$ humedad

En general, se puede apreciar que la zona cafetalera de Jericó es superior en puntos en taza, rendimiento y humedad final lograda. Esto último sin embargo nos indica que la humedad final obtenida se encuentra ligeramente por encima del rango esperado de entre $12 \%$ a $14 \%$ de humedad como lo obtenido por Villegas-Santiago et al. (2020). 
Tabla I. Estadísticos principales resultantes de la toma de muestras

\begin{tabular}{|c|c|c|c|}
\hline Estadístico & Calidad Taza & Rendimiento & Humedad \\
\hline Media & 82,1375 & 72,7370 & 13,8100 \\
\hline Error estándar de la media & $0,0544 I$ & 0,35944 & 0,08350 \\
\hline Mediana & 82,1250 & 72,5050 & 13,8500 \\
\hline Moda & $82,00^{\mathrm{a}}$ & $71,66^{\mathrm{a}}$ & $13,60^{\mathrm{a}}$ \\
\hline Desviación estándar & $0,344 I 4$ & 2,27333 & 0,52808 \\
\hline Varianza & 0,118 & 5,168 & 0,279 \\
\hline Rango & 1,25 & 10,00 & 2,40 \\
\hline Mínimo & 81,50 & 69,00 & 12,30 \\
\hline Máximo & 82,75 & 79,00 & 14,70 \\
\hline a. Existen múltiples modos. Se muestra el valor más pequeño. \\
\hline
\end{tabular}

En Tabla I se aprecia que la calidad en taza es 82, I4, siendo el mínimo 81,50 y máximo 82,75 considerándose entonces café limpio en taza. La humedad lograda promedio es de 13 ,8 $1 \%$ estando dentro del rango esperado, con una desviación estándar y error estándar de la media reducida. Con respecto a lo obtenido por Guevara-Sánchez et al. (2019) quienes lograron valores en taza cercanos a 84.25, el estudio se aleja de esos resultados, asumimos esto a que el tratamiento de café Honey tiene características especiales relacionadas con el tratamiento postcosecha y otros aspectos como la altura de los sitios de donde se obtuvieron las muestras. Así mismo, respecto a la humedad, el dispositivo en promedio obtiene 13,81\% que con respecto a Guevara-Sánchez et al. (2019) es similar.

A continuación, la Figura 4 refleja el promedio general de los valores obtenidos por el total de las 40 muestras de café Honey.

Figura 4. Valores promedio de las características organolépticas de las muestras de café secadas con el

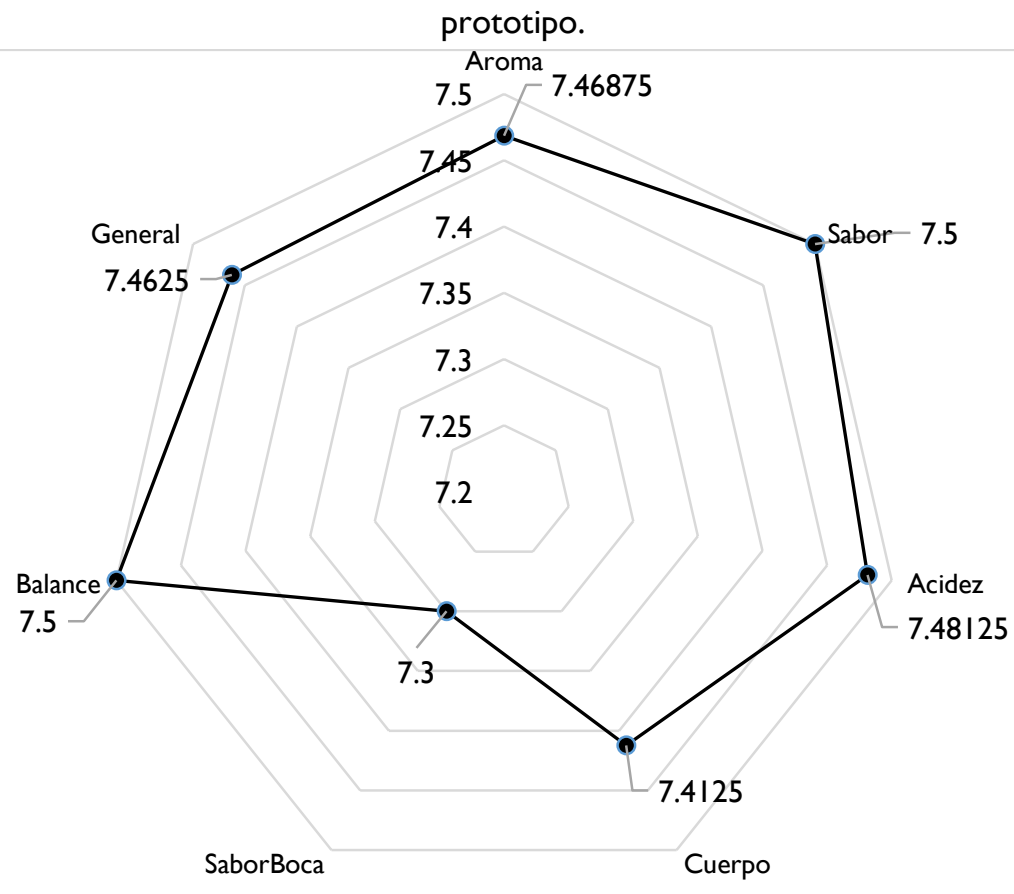


Según la ¡Error! No se encuentra el origen de la referencia., en función a las características organolépticas medidas por el catador en general las muestras son jugosas, radiantes, alanceadas y frescas.

\section{Conclusiones}

El dispositivo construido cuenta con las condiciones necesarias para realizar el proceso de secado en la postcosecha bajo condiciones controladas a través de un mecanismo en invernadero en el que se analizaron 40 muestras, todas ellas no presentaron problemas de fermentación o secado; por lo cual el mecanismo de proceso, garantiza inocuidad y calidad básica para su oferta.

La mayor puntuación registrada en el análisis organoléptico fue de 82.75 puntos en taza, la cual presenta una taza con atributos sensoriales propios de los cafés especiales de alta calidad. Se determina que existe una mejor proyección en la puntuación de hasta 2 puntos, debido a que las muestras se fermentaron sólo 14 horas (se puede fermentar más tiempo en cerezo), el cual le resta calidad sensorial en el análisis. Los rangos de puntuación más altos en el análisis organoléptico se encuentran en el grupo 03, perteneciente a la zona cafetalera Jericó.

Las características principales de perfil de sabor en el grupo 03 predominan, las fragancias dulces a azúcar de caña, caramelo y chocolate, con sabores frutales a mandarina y naranja; con una acidez cítrica brillante, cuerpo suave y jugoso; en general un café con mucho balance; sabores y perfiles propios de cafés de proceso Honey de calidad competitiva. Los rangos de puntuación más bajos en el análisis organoléptico se encuentran en el grupo 01 y 02.

\section{Agradecimientos}

Al Programa Nacional de Desarrollo Tecnológico e Innovación - Prolnnóvate por el financiamiento del proyecto "Desarrollo de un prototipo de secador solar tipo túnel con microclima auto controlado para obtener cafés honey de alta calidad en taza en la provincia de Moyobamba" con Contrato N² 292-INNOVATEPERU-PIEC-2019.

\section{Referencias bibliográficas}

Adonis, M., \& Khan, M. T. E. (2004). Combined convective and infrared drying model for food applications. 2004 IEEE Africon. 7th Africon Conference in Africa, 2, I049-I 052. https://doi.org/I0.I I09/AFRICON.2004.I406850

Alibayan, J. P. I., Bobadilla, I. R. C., Carnicer, M. K. V, Pascua, R. T., Teodosio, J. G., Arago, N. M., Tolentino, L. K. S., Fernandez, E. O., \& Valenzuela, I. C. (2019). Green Coffee Bean Sorter and Corrector based on Moisture Content using Capacitive Method. 2019 IEEE I I th International Conference on Humanoid, Nanotechnology, Information Technology, Communication and Control, Environment, and Management, I-4. https://doi.org/I0.I I09/HNICEM48295.2019.9073477

Arias Escobar, J. P., Zartha Sossa, J. W., Hernández Zarta, R., \& Gómez Garcés, J. (2017). Vigilancia tecnológica y análisis del ciclo de vida de la tecnología: Aplicación en productos generados a partir del café. Revista Electrónica Gestión de Las Personas y Tecnologías, 10(29), 
78-94. https://www.revistas.usach.cl/ojs/index.php/revistagpt/article/view/296I

Barrera, Ó., Gutiérrez, N., \& Orozco-Blanco, D. (2019). Caracterización y diferenciación de cafés, a partir de espectroscopía infrarroja. Revista U.D.C.A Actualidad \&amp; Divulgación Científica, 22. https://doi.org/10.31910/rudca.v22.n1.2019.1158

Bolaños, P. A., Céspedes U, S., \& Cuéllar, J. C. (2018). Prototype of a wireless sensor network for monitoring the coffee drying process. IV School of Systems and Networks. 2178, 6I-63. http://ceur-ws.org/Vol-2178/SSN2018_paper_19.pdf

Borém, F. M., Ribeiro, F. C., Figueiredo, L. P., Giomo, G. S., Fortunato, V. A., \& Isquierdo, E. P. (20I3). Evaluation of the sensory and color quality of coffee beans stored in hermetic packaging. Journal of Stored Products Research, 52, 1-6. https://doi.org//0.1016/j.jspr.2012.08.004

Chunshan, L., Siyu, C., Wenfu, W., Rui, W., \& Hao, Z. (2016). Experimental Study on Heat Transfer Effect of Far Infrared Convection Combined Drying. 2016 International Conference on Intelligent Transportation, Big Data \& Smart City (ICITBS), 505-508. https://doi.org/I0.1 109/ICITBS.2016.38

da Costa, F. O., Alvarenga, T. F., de Mesquita, T. V. C., \& Petri Júnior, I. (202I). Hybrid drying of pulped arabica coffee cherry beans (Coffea arabica L. cv. Catuai) using a hexagonal microwave dryer designed by numerical simulations. Journal of Food Process Engineering, 44(5). https://doi.org/ 0.1 I I l/jfpe. 13666

Díaz Vargas, C., \& Carmen Willems, M. (2017). Línea base del sector café en el Perú. Programa de las Naciones Unidas para el Desarrollo - PNUD.

Estevez, C. L., Bhat, M. G., \& Bray, D. B. (2018). Commodity chains, institutions, and domestic policies of organic and fair trade coffee in Bolivia. Agroecology and Sustainable Food Systems, 42(3), 299-327. https://doi.org/I0.1080/2 I683565.2017.1359737

Guevara-Sánchez, M., Bernales, C., Saavedra-Ramírez, J., \& Owaki-López, J. (2019). Effect of altitude on coffee (Coffea arabica L.) quality: comparison between mechanical and traditional drying. Scientia Agropecuaria, 10(4), 505-510. https://doi.org//0.17268/sci.agropecu.2019.04.07

International Coffee Organization, I. (202I). Historical Data on the Global Coffee Trade. International Coffee Organization. https://www.ico.org/new_historical.asp

Kaveh, M., Abbaspour-Gilandeh, Y., Fatemi, H., \& Chen, G. (202I). Impact of different drying methods on the drying time, energy, and quality of green peas. Journal of Food Processing and Preservation. https://doi.org/I 0.I I I I/jfpp. 15503

Larasati, D. A., Kalandro, G. D., Fibriani, I., Hadi, W., Herdiyanto, D. W., \& Sarwono, C. S. (2018). Optimization of Coffee Bean Drying Using Hybrid Solar Systems and Wi-Fi Data Communication. 2018 International Conference on Electrical Engineering and Computer Science (ICECOS), 29-32. https://doi.org/10.1 I09/ICECOS.2018.8605I 96

Márquez Romero, F., Julca Otiniano, A., Canto Saenz, M., Soplín Villacorta, H., \& Vargas Winstanley, S. (2016). Environmental sustainability in coffee farms after an organic certification process at la convencion (Cusco, Perú). Ecología Aplicada, I5(2), I25-132. http://dx.doi.org//0.21704/rea.v15i2.752 
Prada, Á., Vela, C. P., Bardález, G., \& Saavedra, J. (2019). Effectiveness of a coffee drying process using solar dryers with a continuous air flow system powered by photovoltaic energy, in the San Martín region, Peru . Informacion Tecnologica, 30(6), 85-92. https://doi.org//0.4067/S07/8-07642019000600085

Rodriguez, Y. F. B., Guzman, N. G., \& Hernandez, J. G. (2020). Effect of the postharvest processing method on the biochemical composition and sensory analysis of arabica coffee. Engenharia Agricola, 40(2), I77-183. https://doi.org//0.1590//809-4430ENG.AGRIC.V40N2PI77-I83/2020

Specialty Coffee Association. (2003). Protocols \& Best Practices. Specialty Coffee Association. https://sca.coffee/research/protocols-best-practices

Sotelo-Valer, F., Huamán-Sayán, L., \& Mamani-Arroyo, E. (2020). Design and Implementation of an Automatic Coffee Dryer. 69-73. https://doi.org/I0.I I45/3429536.3429548

Villegas-Santiago, J., Gómez-Navarro, F., Domínguez-Niño, A., García-Alvarado, M. A., SalgadoCervantes, M. A., \& Luna-Solano, G. (2020). Effect of spray-drying conditions on moisture content and particle size of coffee extract in a prototype dryer. Revista Mexicana de Ingeniera Quimica, 19(2), 767-78I. https://doi.org/I0.24275/rmiq/Proc767

Zuluaga-Bedoya, C., \& Gómez, L. M. (20I5). Dynamic modeling of coffee beans dryer. 2015 IEEE 2nd Colombian Conference on Automatic Control (CCAC), I-6.

https://doi.org/I0.1 I09/CCAC.2015.73452I4

\section{Financiamiento}

Programa Nacional de Desarrollo Tecnológico e Innovación - Prolnnóvate que financió del proyecto "Desarrollo de un prototipo de secador solar tipo túnel con microclima auto controlado para obtener cafés honey de alta calidad en taza en la provincia de Moyobamba" con Contrato N²92-INNOVATEPERU-PIECI-2019.

\section{Conflicto de intereses}

El artículo no presenta conflicto de intereses.

\section{Contribución de autores}

Dávila-Guamuro, Jhon: Coordinador general del subproyecto, encargado de su ejecución y del logro de los objetivos. Dio visto bueno y aprobó la versión final del artículo

Llanos-Pérez, Jossy: Asistente de investigación, responsable de la revisión de los informes de avance en la ejecución del proyecto. Dio visto bueno y aprobó la versión final del artículo.

Cabanillas-Pardo, Lenin: Responsable del diseño técnico del dispositivo. Dio visto bueno y aprobó la versión final del artículo. 